\title{
Qualidade nutricional de rações secas para cães adultos comercializadas em Rio Verde - GO
}

\author{
William Germano Carpim ${ }^{1}$ \\ Maria Cristina de Oliveira ${ }^{2 *}$ \\ ${ }^{1}$ Faculdade de Zootecnia, Universidade de Rio Verde \\ ${ }^{2}$ Faculdade de Medicina Veterinária, Universidade de Rio Verde \\ Caixa Postal 244, CEP 75901-970, Rio Verde - GO, Brasil \\ *Autor para correspondência \\ cristina@fesurv.br
}

Submetido em 03/07/2008

Aceito para publicação 11/12/2008

\section{Resumo}

Foi avaliada a qualidade nutricional de rações secas para cães adultos e verificou-se se as informações nos rótulos estavam de acordo com a legislação e com as análises laboratoriais e se o consumo sugerido supria as exigências nutricionais de cães adultos. Foram adquiridas e analisadas amostras de 18 rações comerciais, oito do tipo econômico e 10 premium. Quanto à conformidade com o rótulo, somente 12,5 e 10\% (umidade), 87,5 e 70\% (PB), 25 e 20\% (EEHA), 0 e 0\% (MM), 12,5 e 40\% (Ca) e 0 e 20\% (P) das rações econômicas e premium, respectivamente, estavam conformes. Quanto aos níveis de garantia, a maioria das rações apresentou níveis dentro dos limites. O consumo médio diário sugerido de ração premium foi 13,07\% menor, os consumos de PB e de $\mathrm{P}$ foram 10 e 16,87\% maiores e os consumos de Ca e EM foram 16,27 e 11,15\% menores comparado com o consumo de ração econômica. Concluiu-se que as rações secas para cães adultos, vendidas em Rio Verde, são de boa qualidade já que atendem os limites exigidos pela legislação, há deficiências na rotulagem em relação aos níveis de garantia e há excesso no fornecimento diário de energia com o uso de rações econômicas.

Unitermos: nutrição animal, valor nutritivo, tipo de ração

\section{Abstract}

Nutritional quality of dry food for adult dogs, commercialized in Rio Verde-GO. We evaluated the nutritional quality of dry food for adult dogs, checking whether the label information was in agreement with the law and with the analyses, and also whether the suggested intake reached the nutritional requirements of adult dogs. Eighteen samples of commercial feed, eight of the economical type and 10 of the premium type, were purchased and analyzed. Regarding the product descriptions, only 12.5 and $10 \%$ (moisture), 87.5 and $70 \%(\mathrm{CP}), 25$ and $20 \%$ (AHEE), 0 and $0 \%(\mathrm{MM}), 12.5$ and $40 \%(\mathrm{Ca})$ and 0 and $20 \%(\mathrm{P})$ of the economical and premium foods, respectively, were in conformity according to the label. Regarding the guarantee levels, most of the foods showed levels which were under the limits. The suggested daily average intake of premium food was $13.07 \%$ lower, CP and P intakes were 10 and 16.97\% higher, and Ca and EM intakes were 16.27 and 11.15\% lower compared to the suggested economical food intake. It was concluded that the dry food for adult dogs, sold in Rio Verde, has a good quality because it reaches the required limits, but there are deficiencies in the label information related to the guarantee levels, and there are excesses in the daily energy supply with the use of economical food.

Key words: animal nutrition, food type, nutritive value 


\section{Introdução}

A alimentação é uma das práticas de manejo mais importantes do proprietário de cães e a preocupação nutricional vem sendo reconhecida como parte integrante tanto do cuidado preventivo com a saúde como dos protocolos de tratamento médico e cirúrgico de pacientes.

A indústria da alimentação animal tem evoluído rapidamente e o termo "ração", utilizado para expressar "dieta balanceada" em outras produções animais, vem sendo substituído, neste segmento, por "alimentos completos", por meio da Instrução Normativa $n^{\circ} .9$, de 14 de julho de 2003, que regulamenta os padrões de identidade e qualidade de alimentos completos destinados a cães (MAPA, 2003).

Em 2007, a indústria brasileira produziu 1,8 milhões de toneladas de alimentos para cães e gatos. A Região Sudeste é a responsável por este resultado com $52,64 \%$ do mercado, seguida pela Sul $(40,42 \%)$, Centro-Oeste $(4,45 \%)$, Nordeste $(1,94 \%)$, Norte $(0,55 \%)$ (Anfal-Pet, 2008).

No Brasil, a responsabilidade pela regulamentação das rações para cães e gatos é do Ministério da Agricultura, Pecuária e Abastecimento, prevista no Decreto n ${ }^{\circ} .76 .986$ de 6 de janeiro de 1976. O Decreto é instruído por meio de Instruções Normativas, que são atualizadas e publicadas periodicamente. A Instrução Normativa $\mathrm{n}^{\mathrm{o}}$. 09, publicada em 2007 fixa as características mínimas de qualidade que os alimentos completos e especiais para cães e gatos devem obedecer.

Quanto aos níveis de garantia, existem limites que devem ser obrigatoriamente seguidos pelas indústrias. Segundo MAPA (2003), os valores nutricionais para alimentos secos para cães adultos, em manutenção, devem ser os seguintes: 12\% de umidade (máximo), $16 \%$ de proteína bruta (mínimo), 4,5\% de extrato etéreo (mínimo), 6,5\% de fibra bruta (máximo), 12\% de matéria mineral (máximo), 2,4\% de cálcio (máximo) e $0,6 \%$ de fósforo (mínimo).

Segundo Case et al. (1998), define-se como estado de manutenção a situação do cão que atinge seu tamanho adulto e que não esteja em gestação, lactação ou em trabalho. De acordo com Kienzle e Rainbird (1991), a maioria das raças possui exigência energética de $132 \mathrm{kcal}$ por $\mathrm{kg}$ de $\mathrm{PV}^{0,75} / \mathrm{dia}$, para cães em manutenção e entre cães com pesos entre 5,5 e 54 kg, a exigência energética foi de $126 \mathrm{kcal} / \mathrm{kg}$ de $\mathrm{PV}^{0,75} / \mathrm{dia}$.

Segundo o NRC (1985), as exigências para um cão adulto, em manutenção, são de $5 \%$ de extrato etéreo, $0,59 \%$ de cálcio e $0,44 \%$ de fósforo com base na matéria seca e, para um cão de $10 \mathrm{~kg}, 742 \mathrm{kcal} / \mathrm{d}$ de energia metabolizável. De acordo com Fraser et al. (1996), cães em manutenção devem ingerir, com base em $100 \mathrm{~g}$ de matéria seca, de 20 a $28 \mathrm{~g}$ de $\mathrm{PB}, 10$ a $20 \mathrm{~g}$ de EE, 0 a $5 \mathrm{~g}$ de FB, 0,5 a $0,9 \mathrm{~g}$ de $\mathrm{Ca}$ e 0,4 a $0,9 \mathrm{~g}$ de $\mathrm{P}$.

Assim, esse experimento foi realizado para avaliar a qualidade nutricional das rações secas para cães adultos, de várias marcas, comercializadas em Rio Verde-GO, para verificar se as informações nos rótulos estavam de acordo com a legislação, se eram compatíveis com as análises realizadas e se a quantidade de ração sugerida pelos fabricantes atendia as necessidades nutricionais de cães.

\section{Material e Métodos}

Foram adquiridas, entre os meses de fevereiro e março de 2008, aleatoriamente, 18 marcas comerciais de rações para cães adultos, sendo oito do tipo econômico e dez do tipo premium, fabricadas por nove empresas diferentes e comercializadas em supermercados e pet shops da cidade de Rio Verde-GO.

As amostras foram embaladas em sacos plásticos numerados e enviadas ao laboratório para que análises de proteína bruta $(\mathrm{PB})$, extrato etéreo hidrólise ácida (EEHA), matéria mineral (MM), matéria seca (MS), fibra bruta $(\mathrm{FB})$, cálcio $(\mathrm{Ca})$ e fósforo $(\mathrm{P})$ fossem realizadas segundo a Association of Analytical Communities (Cunnif, 1997).

Depois de obtidos os resultados das análises laboratoriais, foram calculados os teores de energia metabolizável (EM) das rações, por meio da equação $\mathrm{EM}=(\mathrm{PB} \times 35)+(\mathrm{EE} \times 85)+\mathrm{ENN} \times 35)$, sendo os valores de EM expressos em $\mathrm{kcal} / \mathrm{kg}$ de MS. O ENN foi obtido subtraindo-se de 100 os valores de PB, EE, 
FB, MM e umidade e foi expresso em percentagem (Veronesi, 2003).

Os valores determinados nas análises laboratoriais (VD) foram comparados com os valores descritos nos rótulos (VR) pelos fabricantes. A tolerância de 10\% foi adotada na comparação, seguindo a legislação (MAPA, 1976) e o critério utilizado foi de conformidade, expressa em percentagem, para rações cujos resultados das análises laboratoriais estavam de acordo com os valores descritos nos rótulos. Os resultados foram ainda comparados com os valores mínimos e máximos permitidos pelo Ministério da Agricultura, Pecuária e Abastecimento (MAPA, 2003).

A quantidade de ração média a ser fornecida diariamente, sugerida pelas empresas para cada marca, foi também avaliada para verificar se atendia a exigência nutricional de cães com $10 \mathrm{~kg}$ de peso corporal. A exigência diária de energia metabolizável (EM) foi determinada da seguinte forma: $\mathrm{EM}=132 \times \mathrm{PC}^{0,75}$ (Kienzle e Rainbird, 1991; MAPA, 2003), sendo PC o peso corporal.

\section{Resultados e Discussão}

Os valores declarados nos rótulos e os resultados das análises das rações secas para cães adultos em manutenção encontram-se na Tabela 1.

\section{Conformidade com o rótulo}

Segundo o artigo 45 do decreto $\mathrm{n}^{\circ} 76.986$ (MAPA, 1976), os produtos destinados à alimentação animal, segundo os resultados das análises, são classificados: em dentro do padrão, fora do padrão e impróprio para o consumo. São considerados fora de padrão aqueles produtos cujos resultados da respectiva análise apresentem diferenças para mais ou para menos, de 10\% sobre os níveis de garantia aprovados pela Divisão de Nutrição Animal e Agrostologia (DNAGRO).

Na avaliação de conformidade com o rótulo, dentre as rações econômicas avaliadas, $100 \%$ apresentaram níveis de EEHA, FB, MM e P compatíveis com os valores declarados nos rótulos e quantos aos teores de

TABELA 1: Composição média das rações secas com base nos valores médios declarados nos rótulos (VR) e nos determinados nas análises laboratoriais (VD).

\begin{tabular}{|c|c|c|c|c|c|}
\hline \multirow{2}{*}{ Parâmetro } & \multirow{2}{*}{ Ração* } & \multirow{2}{*}{ VR } & \multirow{2}{*}{ VD } & \multicolumn{2}{|c|}{ Conformidade (\%) } \\
\hline & & & & com VR & com LPL \\
\hline \multirow{2}{*}{ Umidade $(\%)$} & Econômica & $11,75 \pm 0,34$ & $9,60 \pm 0,37$ & 12,50 & 100,00 \\
\hline & Premium & $11,30 \pm 0,31$ & $8,75 \pm 0,33$ & 10,00 & 100,00 \\
\hline \multirow{2}{*}{ PB (\%) } & Econômica & $18,37 \pm 0,59$ & $20,61 \pm 1,07$ & 87,50 & 100,00 \\
\hline & Premium & $23,20 \pm 0,53$ & $24,97 \pm 0,95$ & 70,00 & 100,00 \\
\hline \multirow{2}{*}{ EEHA $(\%)$} & Econômica & $6,31 \pm 0,66$ & $9,05 \pm 0,82$ & 25,00 & 100,00 \\
\hline & Premium & $9,87 \pm 0,59$ & $10,67 \pm 0,73$ & 20,00 & 100,00 \\
\hline \multirow{2}{*}{ FB $(\%)$} & Econômica & $6,06 \pm 0,30$ & $4,38 \pm 0,47$ & 0,00 & 100,00 \\
\hline & Premium & $3,60 \pm 0,27$ & $5,08 \pm 0,42$ & 0,00 & 90,00 \\
\hline \multirow{2}{*}{ MM (\%) } & Econômica & $11,62 \pm 0,58$ & $8,87 \pm 0,95$ & 0,00 & 100,00 \\
\hline & Premium & $8,98 \pm 0,52$ & $8,68 \pm 0,85$ & 10,00 & 80,00 \\
\hline \multirow{2}{*}{$\mathrm{Ca}(\%)$} & Econômica & $2,37 \pm 0,11$ & $2,26 \pm 0,22$ & 12,50 & 37,50 \\
\hline & Premium & $1,92 \pm 0,10$ & $2,10 \pm 0,20$ & 40,00 & 70,00 \\
\hline \multirow[t]{2}{*}{$\mathrm{P}(\%)$} & Econômica & $0,66 \pm 0,04$ & $1,25 \pm 0,10$ & 0,00 & 100,00 \\
\hline & Premium & $0,89 \pm 0,03$ & $1,15 \pm 0,09$ & 20,00 & 100,00 \\
\hline \multirow[t]{2}{*}{ Relação Ca:P } & Econômica & $3,70 \pm 0,18$ & $1,75 \pm 0,12$ & - & - \\
\hline & Premium & $2,13 \pm 0,16$ & $1,84 \pm 0,10$ & - & - \\
\hline \multirow[t]{2}{*}{ EM (kcal/kg) } & Econômica & $2818 \pm 60$ & $3155 \pm 44$ & - & - \\
\hline & Premium & $3158 \pm 53$ & $3246 \pm 39$ & - & - \\
\hline
\end{tabular}

$\mathrm{VD}=$ valor determinado $\mathrm{VR}=$ valor do rótulo $\mathrm{LPL}=$ limites permitidos por lei

*Foram utilizadas 10 amostras do tipo premium e oito amostras do tipo econômico. 
umidade, $\mathrm{PB}$ e Ca, $12,50,87,50$ e 12,50\% das amostras estavam em conformidade com os rótulos.

Dentre as do segmento premium, $100 \%$ delas tiveram níveis de $\mathrm{FB}, \mathrm{MM}$ e $\mathrm{P}$ dentro dos limites de $10 \%$ acima ou abaixo dos declarados nos rótulos. Quanto aos teores de umidade, PB, EEHA, MM, Ca e P, 10, 70, $20,10,40$ e $20 \%$ das amostras avaliadas apresentaram valores em conformidade com os rótulos.

De acordo com o Código de Defesa do Consumidor, artigo $6^{\circ}$ da Lei ${ }^{\circ} 8078$, de 11/09/1990, é direito básico do consumidor o acesso à informação adequada e clara sobre os diferentes produtos e serviços, com especificações claras sobre quantidade, características, composição, qualidade e preço, bem como sobre os riscos que apresentem (CDC, 1990).

Os níveis de garantia estabelecem a qualidade nutricional do produto que está sendo oferecido ao consumidor, garantindo um padrão de qualidade que depende um controle adequado do processo produtivo e da matéria-prima utilizada. O consumidor muitas vezes considera um produto melhor que outro baseado nas informações do rótulo e, no caso das informações não serem corretas, o consumidor está sendo induzido a erro. Além disso, as classificações de produto econômico ou premium podem ser levadas em consideração pelo consumidor no momento da compra.

\section{Conformidade com os limites permiti- dos por lei}

Todas as amostras de rações do segmento econômico avaliadas estavam em conformidade com os limites permitidos pela Instrução Normativa ${ }^{\circ} 9$ de 2003 (MAPA, 2003), com exceção do teor de cálcio, em que somente $37,50 \%$ das amostras apresentaram valores iguais ou inferiores a 2,40\% de cálcio (Tabela 1). Com relação às rações do tipo premium, $100 \%$ das amostras estavam em conformidade com a legislação quanto aos teores de umidade, proteína bruta, extrato etéreo e fósforo, porém, $10 \%$ delas continham teores de fibra bruta superiores a 6,50\%, 20\% apresentaram teores de MM superiores a $12 \%$ e $30 \%$ tiveram níveis de cálcio superiores a $2,40 \%$, que são os valores máximos permitidos pelo Ministério da Agricultura, Pecuária e Abastecimento.
Carciofi et al. (2006) observaram elevada concentração de fibra nos produtos econômicos $(6,3 \%)$ e, segundo os autores esses produtos contêm farelos vegetais ricos em fibra. Entretanto, nesse experimento foi observado elevado teor de FB em 10\% das rações premium, provavelmente pela presença de farelo de trigo $(9,66 \%)$ e de soja $(5,41 \%)$.

Burrows et al. (1982) utilizaram dietas contendo $0,6,6,5,11,2$ e $14,7 \%$ de fibra para cães e notaram que, na medida em que o teor de fibra dietética aumentou, houve redução na digestibilidade da matéria seca e aumento no peso e no teor de umidade das fezes dos animais, além de aumentar o trânsito intestinal de 39,4 horas com a dieta com $0,6 \%$ para 28,7 horas para a dieta com $14,7 \%$.

As fibras dietéticas podem ser benéficas à saúde dos cães. Os ácidos graxos voláteis (AGV) produzidos pela fermentação bacteriana das fibras solúveis no cólon canino promovem maior absorção de água e de eletrólitos já que os AGV estimulam a proliferação dos enterócitos (Donatto et al., 2006), aumentando a superfície de absorção intestinal. Há, entretanto outros efeitos, como os descritos por Burkhalter et al. (2001) que estudaram a inclusão de níveis de fibra (4,8 a 7,3\%) na dieta de cães e observaram que o consumo de ração aumentou com a inclusão de maiores níveis de FB como conseqüência da menor digestibilidade da MS, MO e da EB. Além disso, o peso das fezes aumentou de 53 para $67 \mathrm{~g}$ de MS/dia.

O excesso de MM nas ração do tipo premium pode ser conseqüência do tipo de fonte protéica utilizada na formulação das rações. Ingredientes muito comuns em rações para cães, como a farinha de carne, de carne e ossos e de vísceras de frango possuem elevados teores de minerais. A farinha de carne possui $28,8 \%$ (NRC, 1985), a farinha de vísceras possui $15 \%$ e a farinha de carne e ossos possui de 21,76 a 42\% de MM (Rostagno et al., 2005). Minerais em excesso comprometem a qualidade das rações, pois quanto maior o teor de matéria mineral, geralmente representada pela maior participação da farinha de carne e ossos na composição do ingrediente, menor sua digestibilidade já que haverá redução no teor de matéria orgânica do alimento. Além disso, essas farinhas protéicas possuem grande quantidade de $\mathrm{Ca}$ e $\mathrm{P}$ (Carciofi et al., 2006). 
A elevada ingestão de cálcio não é benéfica aos animais, pois o excesso desse mineral resulta em competição e redução da absorção intestinal de outros minerais, principalmente fósforo, zinco e manganês (Schoulten, 2002). A alta concentração de cálcio em algumas rações, principalmente as utilizadas na alimentação de grande porte, pode resultar em maior incidência de doenças osteoarticulares, pois a hipercalcemia diminui a taxa de remodelagem óssea ocasionando, muitas vezes, má formação óssea (Fau et al., 2005), além de induzir ao hipoparatireoidismo secundário (Yamamoto et al., 1995). Dietas contendo níveis elevados de $\mathrm{Ca}(3,3 \%)$ ou reduzidos $(0,55 \%)$ induzem distúrbios ósseos durante a fase de crescimento que podem perdurar durante a vida adulta (Weber et al., 2000).

De acordo com Schoenmakers et al. (1999), cães alimentados com dietas ricas em $\mathrm{Ca}$, com ou sem aumento proporcional de $\mathrm{P}$, após o desmame tiveram maiores taxas de retenção de $\mathrm{Ca}$ do que aqueles que consumiram dietas com teor recomendado de $\mathrm{Ca}$. Quando já adultos, e após longo período de adaptação, a retenção de Ca não diferiu dos que receberam dietas com teores normais de $\mathrm{Ca}$. Isso ocorre devido a redução na absorção de $\mathrm{Ca}$ e, como conseqüência, a absorção de $\mathrm{P}$ também diminuiu, com absorção desproporcional de $\mathrm{Ca}$ e $\mathrm{P}$ nos animais dos grupos rico em $\mathrm{Ca}$, mas com $\mathrm{P}$ normal.

A relação Ca:P média das rações avaliadas (Tabela 1) foram 1,75:1 no segmento econômico e 1,84:1 no premium. É importante a manutenção de adequada relação Ca:P (1:1 a 2:1, no máximo), pois o excesso de um dos minerais compromete a absorção do outro. Hazewinkel et al. (1991) demonstraram que dietas com relações $\mathrm{Ca}: \mathrm{P}$ elevadas promoveram menor consumo de ração e reduziram o crescimento de cães.

O consumo diário de nutrientes e de EM das rações secas para cães adultos, encontram-se na Tabela 2 .

Embora a quantidade média de ração premium sugerida pelos fabricantes para consumo por um cão de $10 \mathrm{~kg}$ de peso corporal seja $13,07 \%$ menor em relação a quantidade recomendada para rações econômicas, as variações nos teores de $\mathrm{EE}$ e $\mathrm{FB}$ foram muito pequenas. Entretanto, o consumo de PB aumenta em $10 \%$ e o de P
TABELA 2: Consumo diário de nutrientes e de energia metabolizável das rações secas para cães adultos, com base na matéria seca.

\begin{tabular}{lcc}
\hline \multirow{2}{*}{} & \multicolumn{2}{|c}{ Rações } \\
\cline { 2 - 3 } & Econômica & Premium \\
\hline Consumo de MS (g/d) & $235 \pm 15$ & $206 \pm 14$ \\
Consumo de PB (g/d) & $50,45 \pm 3,91$ & $55,50 \pm 3,50$ \\
Consumo de EE (g/d) & $23,53 \pm 1,67$ & $23,31 \pm 1,50$ \\
Consumo de FB (g/d) & $11,35 \pm 0,80$ & $11,28 \pm 1,20$ \\
Consumo de Ca (g/d) & $5,90 \pm 0,77$ & $4,94 \pm 0,68$ \\
Consumo de P (g/d) & $3,26 \pm 1,55$ & $4,51 \pm 1,39$ \\
Consumo ENN (g/d) & $115,29 \pm 9,85$ & $85,54 \pm 8,81$ \\
Consumo de EM (kcal/d) & $780 \pm 52$ & $693 \pm 46$ \\
\hline
\end{tabular}

* considerando um consumo sugerido na embalagem, com base na MN, de 260 e $226 \mathrm{~g} / \mathrm{d}$ para as rações econômicas e premium, respectivamente, para um cão adulto de $10 \mathrm{~kg}$.

em $16,87 \%$ e o consumo de Ca diminui em $16,27 \%$ e o de EM em 11,15\%. Os valores obtidos, entretanto, estão de acordo com os relacionados por Fraser et al. (1996).

A densidade energética da dieta deve ser suficientemente alta para permitir que os cães obtenham calorias suficientes para manter o balanço energético, já que a energia é o principal fator que determina a quantidade de alimento consumido por dia e, portanto, a ingestão dos demais nutrientes. Segundo MAPA (2003), as necessidades de EM diárias de um cão com $10 \mathrm{~kg}$ é de $742 \mathrm{kcal}$. Considerando esse valor como referência, as rações do segmento econômico estariam fornecendo calorias em excesso, predispondo o animal à obesidade.

Muitos proprietários alimentam os animais de estimação à vontade com um produto seco e não se preocupam com a sobrecarga no consumo. Entretanto, com a fabricação de produtos de alta qualidade, alta palatabilidade e alta densidade energética, uma menor quantidade do produto é necessária para alimentar os animais (Case et al., 1998).

As rações econômicas para cães não costumam ser formuladas para atender os diferentes estágios da vida do cão. Nesse sentido, é importante ressaltar que as necessidades energéticas do cão variam com sua idade. 
Na medida em que o cão envelhece, suas exigências energéticas diminuem (Finke, 1994) e, caso o animal seja alimentado sempre com a mesma ração na mesma quantidade, é possível que se torne obeso quando se tornar mais velho pela ingestão excessiva de calorias.

Pode-se concluir que a qualidade das rações secas para cães adultos, comercializadas em Rio Verde-GO, são de boa qualidade já que atendem a maioria dos limites máximos e mínimos exigidos pela legislação vigente. Entretanto, há deficiências na rotulagem em relação às informações fornecidas ao consumidor sobre os níveis de garantia dos produtos. Quanto à quantidade de ração consumida diariamente, deve-se considerar a quantidade sugerida como uma estimativa e modificá-la com base em avaliações contínuas do peso corporal e estágio fisiológico do animal.

\section{Referências}

Anfal-Pet - Associação Nacional dos Fabricantes de Alimentos para Animais de Estimação. 2008. Setor de Pet Food (alimentos para animais de companhia) deve faturar US\$ 3,22 bilhões em 2008. Disponível em $<$ http://anfalpet.org.br/Site/principal.php?id menu $=6>$. Acesso em 02 de junho de 2008 .

Burkhalter,T.M.; Merchen, N. R.;Bauer,L.L.; Murray, S.M.; Patil,A. R.;BrentJr., J.L.;Fahey Jr., G.C.2001. Theratioofinsolubleto soluble fiber components in soybean hulls affects ileal and total-tract nutrient digestibilities and fecal characteristics of dogs. Journal of Nutrition, 131(7): 1978-1985.

Burrows, C. F.; Kronfeld, D. S.; Banta, C. A.; Merritt, A. M. 1982. Effects of fiber on digestbility and transit time in dogs. Journal of Nutrition, 112 (9): 1726-1732.

Carciofi, A. C.; Vasconcellos, R. S.; Borges, N. C.; Moro, J. V.; Prada, F.; Fraga, V. O. 2006. Composição nutricional e avaliação de rótulo de rações secas para cães comercialzadas em Jaboticabal-SP. Arquivo Brasileiro de Medicina Veterinária e Zootecnia, 58 (3): 421-426.

Case, L. P.; Carey, D. P.; Hirakawa, D. A. 1998. Nutrição canina e felina. Harcourt Brace de España S.A., Madrid, Espana, 424pp.

CDC - Código de Defesa do Consumidor. Lei no 8.078, de 11 de Setembro de 1990. Diário Oficial da República Federativa do Brasil, em 12/09/1990.

Cunnif, P. 1997. Official methods of analysis of AOAC International. $16^{\text {th }}$ ed. AOAC Gaithersburg, USA, p.1-45.
Donatto, F. F.; Pallanch, A.; Cavaglieri, C. R. 2006. Fibras dietéticas: Efeitos terapêuticos e no exercício. Saúde em Revista, 8 (20): 65 71.

Fau, D.; Remy, D.; Viguier, E.; Carozzo, C.; Chanoit, G.; Genevois, J. P. 2005. Alimentation et dysplasie coxo-fémorale. Revue de Médicine Veterinaire, 156 (3): 138-147.

Finke, M. D. 1994. Energy requirements of adult female Beagles. Journal of Nutrition, 124 (12): 2604S-2608S.

Fraser, C. M.; Bergeron, J. A.; Mays, A.; Aiello, S. E. 1996. Manual Merck de Veterinária. $7^{\mathrm{a}}$ ed. Roca, São Paulo, Brasil, 2169pp.

Hazewinkel, H. A. W.; van den Brom, W. E.; Klooster, A. T. V. T.; Voorhout, G.; van Wees, A. 1991. Calcium metabolism in Great Dane dogs fed diets with various calcium and phosphorus levels. Journal of Nutrition, 121 (11): S99-S106.

Kienzle, E.; Rainbird, A. 1991. Maintenance energy requirement of dogs: what is the correct value for the calculation of metabolic body weight in dogs? Journal of Nutrition, 121 (11): S39-S40.

MAPA - Ministério da Agricultura, Pecuária e Abastecimento. 1976. Decreto $\mathrm{n}^{\circ} 76.986$, de 06 de janeiro de 1976. Diário Oficial da República Federativa do Brasil, Seção 1, p. 499.

MAPA - Ministério da Agricultura, Pecuária e Abastecimento. 2003. Instrução Normativa ${ }^{\circ}$. 9 , de 09 de julho de 2003. Diário Oficial da República Federativa do Brasil, Seção 1, p. 7.

NRC. 1985. Nutrient Requirements of Dogs. National Academy Press, Washington, USA, 88pp.

Rostagno, H. S.; Albino, L. F. T.; Donzele, J. L.; Gomes, P. C.; Oliveira, R. F.; Lopes, D. C.; Ferreira, A. S.; Barreto, S. T. 2005. Tabelas brasileiras para aves e suínos. Composição de alimentos e exigências nutricionais. $2^{\mathrm{a}}$ ed., UFV, Viçosa, Brasil, 186pp.

Schoenmakers, I.; Hazewinkel, H. A. W.; van den Brom, W. E. 1999. Excessive $\mathrm{Ca}$ and $\mathrm{P}$ intake during early maturation in dogs alters $\mathrm{Ca}$ and $\mathrm{P}$ balance without long-term effects after dietary normalization. Journal of Nutrition, 129 (5): 1068-1074.

Schoulten, N. A.; Teixeira, A. S.; Bertechini, A. G.; Freitas, R. T. F.; Conte, A. J.; Silva, H. O. 2002. Efeito dos níveis de cálcio sobre a absorção de minerais em dietas iniciais para frangos de corte suplementadas com fitase. Ciência e Agrotecnologia, 26 (6): 13131321.

Veronesi, C. 2003. Efeito de dois alimentos comerciais secos no consumo energético, peso vivo e peso metabólico, escore corporal, escore e peso fecal de cães adultos em manutenção e atividade. Dissertação de Mestrado, Universidade de São Paulo, Brasil, 93pp.

Weber, M.; Martin, L.; Dumon, H.; Biourge, V.; Nguyen, P. 2000. Growth and skeletal development in two large breeds fed 2 calcium levels. Proceedings of ACVIM FÓRUM, Seattle, USA, CD Rom.

Yamamoto, M.; Seedor, J. G.; Rodan, G. A.; Balena, R. 1995. Endogenous calcitonin attenuates parathyroid hormone-induced cancellous bone loss in the rat. Endocrinology, 136 (2): 788-795. 